\title{
Indolent Non-Hodgkin Lymphoma
}

National Cancer Institute

\section{Source}

National Cancer Institute. Indolent Non-Hodgkin Lymphoma. NCI Thesaurus. Code C8504.

A non-Hodgkin lymphoma with an indolent clinical course. Representative examples include grade 1 and grade 2 follicular lymphoma, marginal zone lymphoma, and lymphoplasmacytic lymphoma. 